\title{
Comunidad de aprendizaje entre mujeres adolescentes
}

\section{Learning Communities for Teenage Women}

\section{Comunidades de aprendizagem para mulheres adolescentes}

\author{
María de Lourdes Martínez Ortiz¹, Gandy Griselda Quijano \\ Zavala $^{2}$ y Alma Delia Sánchez Rivero ${ }^{3}$
}

Fecha de recepción: 08/09/2018

Fecha de evaluación: 10/10/2018

Fecha de aceptación: 25/10/2018

Disponible en línea:

DOI: https://doi.org/10.18359/reds.4363

Cómo citar este artículo:

Martínez, M. L., Quijano, G. G. y Sánchez, A. D. (2018). Comunidad de aprendizaje entre mujeres adolescentes. Revista Educación y Desarrollo Social, 12(2), 85-100.

\footnotetext{
$1 \quad$ Licenciada en Pedagogía de la Universidad Autónoma del Carmen y maestra en Investigación Educativa de la Universidad de Guanajuato.

$2 \quad$ Licenciada en Lengua Extranjera (inglés) de la Universidad Autónoma del Carmen, maestra en Lingüística Aplicada de la Universidad de Ohio y doctora en Lingüística Aplicada de la Universidad de Southampton.

3 Licenciada en Lingüística y Literatura de la Universidad Autónoma de Yucatán, maestra en Antropología Social, opción en Etnohistoria, de la Universidad Autónoma de Yucatán, y doctora en Lenguas y Culturas Románicas de la Universidad Autónoma de Barcelona.
} 


\title{
Resumen
}

En este artículo se presentan los resultados de una intervención que procuró potenciar en mujeres adolescentes procesos reflexivos en torno a sus roles tradicionales, circunstancias contextuales y la importancia de un proyecto de vida. La modalidad bajo la cual se trabajó fue la de taller, permeando elementos constructivistas. De este modo, las mujeres adolescentes se aproximaron a su forma de pensar, sentir y actuar; compartieron entre sí experiencias personales, y reflexionaron sobre cómo se ha ido formando la persona que son, sobre la importancia de repensarse de otro modo, la vitalidad de los propios sueños y la generación de estrategias para materializarlos y reorientarlos.

Palabras clave: aprendizaje; comunidad; mujeres adolescentes.

\begin{abstract}
This article discusses the results of an intervention for fostering reflective practices in adolescent women regarding their traditional roles and contextual circumstances and the importance of establishing a life project. This project was developed through workshops rooted in elements of constructivism. In this way, participating adolescent women were able to approach their way of thinking, feeling, and acting, sharing personal experiences and reflecting on becoming the person they are today, the importance of reinventing themselves, the vividness of their own dreams, and the generation of strategies for materializing and pivoting them.
\end{abstract}

Keywords: adolescent women; community; learning

\section{Resumo}

Este artigo discute os resultados de uma intervenção para fomentar práticas reflexivas em mulheres adolescentes sobre seus papéis tradicionais, circunstâncias contextuais e a importância de estabelecer um projeto de vida. Este projeto foi desenvolvido por meio de oficinas enraizadas em elementos do construtivismo. Dessa forma, as adolescentes participantes puderam abordar seu modo de pensar, sentir e agir, compartilhar experiências pessoais e refletir sobre como se tornaram a pessoa que são hoje, a importância de se reinventar, a vivacidade de seus próprios sonhos e a geração de estratégias para materializá-los e articulá-los.

Palavras-chave: aprendizagem, comunidade, mulheres adolescentes. 


\section{Introducción}

La presente experiencia es resultado del trabajo realizado con un grupo de seis mujeres adolescentes en una localidad semi-urbana, en el municipio de Paraíso, Tabasco, México; a partir de procesos reflexivos respecto a sus roles tradicionales y circunstancias contextuales, estas mujeres fueron capaces de aproximarse a su forma de pensar, de sentir y de actuar. Las participantes pudieron compartir entre sí experiencias personales, su historia, y la manera en cómo se han construido las personas que son actualmente. Se sensibilizó a las mujeres sobre la importancia de repensarse, la vitalidad de sus propios sueños y la generación de estrategias para materializarlos y reorientarlos. El enfoque metodológico fue de comunidad de aprendizaje (CA) y la modalidad de taller. A continuación, se comparte la estructura y desarrollo de la cA, los resultados obtenidos y las respectivas recomendaciones.

\section{Desarrollo}

La localidad en la que se realizó la intervención es un lugar en el que los pobladores se conocen entre sí, y en su gran mayoría son de escasos recursos; no obstante, son personas sencillas y muy trabajadoras. Las mujeres adolescentes participan en las labores domésticas de sus casas o de otras familias, cuidan de sus hermanos menores y algunas logran terminar la secundaria. La mayoría se casan y tienen hijos a muy temprana edad.
Estos antecedentes gestaron la inquietud de una comunidad de aprendizaje (CA), que beneficiara a mujeres adolescentes de la localidad, bajo una propuesta educativa solidaria. Finalmente se tradujo en una experiencia trascendental, significativa, transformadora y con un alto sentido ético-social por todo lo que compartieron las partes involucradas. Al respecto, Flores (2012) manifiesta que:

en la propuesta de comunidad de aprendizaje se reconoce que existe un elemento fundamental que cambia el rol de quien interviene pedagógicamente, y este es la implicación de los sujetos en la identificación misma de sus necesidades e intereses, desde su experiencia de vida y por tanto en la definición de los contenidos del proceso educativo. (p. 6)

La experiencia de la cA se recupera y explicita en el presente escrito con el propósito de fortalecer la premisa de que la formación de un docente puede provenir de diferentes campos y que no tendría sentido si los elementos teóricos no se contrastan y validan en contextos concretos, es decir, en la práctica. Como profesoras, es necesario de incursionar en otras dimensiones, e incluso correr riesgos para seguir aprendiendo, pues de esta manera se obtienen elementos que auto-enriquecen la práctica educativo-formativa propia y la de aquellas personas con quienes se comparte la experiencia.

En este sentido y para esta investigación se propuso desarrollar el objetivo de "formar una comunidad de aprendizaje (CA) 
entre un grupo de mujeres adolescentes, con la intención principal de potenciar entre los participantes, procesos reflexivos en torno a sus roles tradicionales, sus circunstancias contextuales y su proyecto de vida.

\section{Referentes teórico-conceptuales}

\section{Modelo de formación}

En la presente intervención de $\mathrm{CA}$, se propugnó para que los procesos que se dieron al interior de la experiencia formativa con las adolescentes, y retomando a Freire (1994), no se les redujera a ser simplemente espectadoras del proceso, sino que se les consideró como personas activas, responsables y autoras de su propia formación.

La caracterización del proceso registró, en primer lugar, la relación entre las adolescentes y los coordinadores de la CA, quienes promovieron un acercamiento "no ya educador del educando; no ya educando del educador, sino educador-educando con educando-educador" (Freire, 1999, p. 86), por lo que propició que los participantes estuvieron en las mismas posibilidades de interactuar entre sí, a un mismo nivel, dejando a un lado la autoridad o el dominio del educador sobre el educando.

En segundo lugar, el aprendizaje se visualizó como una experiencia en el 88 que de manera conjunta las participantes construyen conocimiento y eso mismo permitió comprender, por un lado, que la interacción y el grupo son un medio y fuente de experiencias que posibilitan el aprendizaje; y, por otro, que el conocimiento no es algo que ya esté dado, sino que por el contrario es relativo y por lo mismo no está terminado.

Y tercero, se asumió que "nadie educa a nadie, así como tampoco nadie se educa a sí mismo, los hombres se educan en comunión, y el mundo es el mediador" (Freire, 1999, p. 86). En suma, la formación se entendió como un "proceso en espiral, donde los aprendizajes que poseen los participantes se reconozcan como la base y punto de partida sobre la que se construyen otros" (Pansza, Pérez y Morán, 1992, p. 85).

Propiciar una experiencia de esta naturaleza no puede considerarse como algo que no enfrentase múltiples obstáculos por las propias personas involucradas; sin embargo, este fue uno de los principales retos, pues resultó necesario "reemplazar el concepto de un educador [...] e incluso de varios educadores [...] por el de un equipo de educadores unido y coherente" (Basis, 1996, p. 117). De igual modo, se sustituyeron los contenidos predeterminados por aquellos que guardaban estrecha relación con las problemáticas, necesidades, intereses y expectativas que les fueron propias a las participantes, y al mismo tiempo estableciendo conexiones con su entorno social.

Bajo tal perspectiva, se retomó el modelo hermeneútico-reflexivo, pues esta perspectiva, se perfila como una práctica 
crítica, participativa, integral, contextualizada, significativa y que como tal se sitúa en el otro extremo y de cara a las que se sostienen en otros modelos de formación, tales como el práctico-artesanal, academicista y tecnicista-eficientista (De Lella, 2003); no obstante, el intentar transitar a ejercicios de formación bajo un modelo hermenéutico-reflexivo se constituyó un proceso complejo. Sin embargo, como parte de las pretensiones de la CA, las dinámicas generadas en cada una de las sesiones propugnaron para que las adolescentes pudiesen:

- Referir sobre su historia y su cosmovisión.

- Identificar formas predominantes de aprender.

- Vivenciar situaciones problematizadoras.

- Conocer el grado o grados de apertura, participación, sensibilidad y compromiso que poseían hasta ese momento de sus vidas.

- Efectuar lecturas de su realidad.

- Identificar roles legitimados e introyectados.

- Poner en práctica el diálogo, la reflexión y las relaciones interpersonales.

\section{Proyecto de vida}

Para hacer referencia a lo que es un proyecto de vida se retomó el Manual de Proyecto de Vida Completo, en el que se alude a toda una parte conceptual sobre proyecto de vida, carrera y planeación (anticipación y decisión). En dicho documento se describen también una serie de pasos para la generación del proyecto, tales como autoconocimiento, actitud de aprendizaje, misión personal, formular objetivos y formular estrategias (Ávila, 2009).

\section{Comunidad de aprendizaje}

La comunidad de aprendizaje (CA), de acuerdo con Berlanga (2005) se concibe como "una estrategia educativa, por lo tanto, se traduce como un espacio de relaciones entre personas que comparten sentidos y significados de mundo de vida, reunidos a partir de intereses compartidos para resolver necesidades de aprendizaje que den lugar a una práctica intencionada de transformación de su realidad".

Esta acepción de CA, resultó adecuada para la intervención, con lo observado en las conductas de las adolescentes, resultaba más que evidente que los patrones de "mujer adolescente" se encontraban naturalizados, determinaban su estilo de vida y condicionaban sus formas de ver y estar en su realidad inmediata.

\section{Estrategia metodológica}

Cabe señalar, que la estrategia bajo la cual se trabajó la comunidad de aprendizaje (CA) fue la de taller, ya que a través de ella es posible

generar aprendizaje significativo tanto individual como colectivamente. Esto implica que se dan cambios de 
actitud, conocimientos y emociones en las personas al respecto de algo, llámese problemas teóricos, prácticos o sociales. Se aprehende haciendo y en el hacer se resuelven problemas que redundan en la transformación de la realidad y por ende la realidad humana, de ahí el aprendizaje significativo. (Sosa, 2002, p. 9)

De esta manera, fue posible traer a flote y poner en juego actitudes, conocimientos y emociones por parte de las adolescentes participantes.

El soporte teórico implícito del taller lo constituyó también, el enfoque constructivista, ya que desde esta postura se concibe al aprendizaje como una función integradora, que permite a las personas construir sus propios conceptos a partir de los anteriores, en el hacer, reflexionar, opinar, sentir, decidir y proponer soluciones. En esta perspectiva también se reconoce que "el conocimiento es una construcción subjetiva en la que la realidad deja de ser una entidad absoluta, como entidad independiente o externa a nosotros mismos" (Cubero, 2005, p. 16). De allí que se entendiera que las participantes en sí mismas resultasen tan valiosas, así como sus pensamientos, experiencias, conocimientos, sentimientos, creencias, tanto que, sin cada uno de estos elementos hubiese sido imposible darle contenido y desarrollar el taller.

Así mismo como parte de la metodología del taller, se hizo una constante problematización del conocimiento. De tal modo que, en el proceso, invariablemente, se planteaban cuestionamientos y las participantes al intentar resolverlos, desarrollaban las temáticas.

Particularmente, como una forma de ir marcando los momentos fundamentales y graduar los avances al interior de la CA se retomó la propuesta metodológica de Gómez (s.f.).

\section{Pre-etapas de la CA}

En el marco de esta propuesta se trabajaron dos etapas previas a la ejecución del proyecto. La primera relacionada con la sensibilización en la que se hizo la participación de todas las participantes y se dio una explicación general del proyecto de comunidad de aprendizaje. Un segundo momento se recuperó entre las participantes los significados de comunidad, aprendizaje y comunidad de aprendizaje.

\section{a) La sensibilización}

En este primer momento tuvo lugar: la presentación de todas las participantes y una explicación general del proyecto de CA.

\section{b) Toma de decisión}

En esta etapa la intervención se circunscribió a la recuperación entre las participantes acerca de ¿qué significamos por comunidad y aprendizaje?, ¿qué es una comunidad de aprendizaje?

\section{Etapas de la CA}

\section{a) El sueño}

En esta etapa se condujo a las participantes a intercambiar experiencias 
y aspiraciones sobre: ¿cuáles son las cosas que me gustaría hacer en la vida?, ¿pienso que puedo lograrlas?, ¿cómo es un día de mi vida?

\section{b) Selección de necesidades}

Recuperando y tomando como pre-textos las aportaciones compartidas por las participantes en la etapa anterior, se les invitó a reflexionar acerca de ¿qué es un proyecto de vida?, ¿qué implica un proyecto de vida?, ¿qué es ser mujer o qué significa para mi ser mujer?, ¿qué tipo de mujeres queremos ser?, ¿podríamos intentar ser diferentes?

\section{c) Otras prioridades}

Al cierre de la experiencia se exhortó a las participantes a rememorar el proceso vivido en con sus familias.

\section{Aspectos pedagógicos}

En la propuesta se aspiró a propiciar un ambiente en el que las adolescentes participantes se sintieran en confianza para externar su pensar y su sentir, independientemente del punto desde el cual se consideraran convocadas; a la par, las otras adolescentes tendrían que disponerse a escuchar, para posteriormente ascender a un ambiente de diálogo, en donde la motivación, sus saberes y experiencias fuesen el discurso que como cualquier otro tiene su justo valor y, por lo tanto, merece ser recuperado, compartido y escuchado.

En este sentido, y retomando las ideas respecto a una CA, se partió de la premisa de que "cada persona y cada miembro de la comunidad es potencialmente un educador y un educando, con capacidad tanto para enseñar como para aprender" (Torres, 1999, p. 2).

Para lograr lo anterior, se procuró que la participación y actitud de las mujeres adolescentes se caracterizara por mostrar apertura al diálogo, al compromiso, al respeto, a la cooperación, a la solidaridad, a no temer a soñar, a equivocarse, a la responsabilidad, que confiaran en sus propias capacidades, saberes, experiencias y en las de las otras. Que cada miembro de la ca pudiese asumirse como plantea Freire como seres de transformación y no de adaptación.

\section{Resultados obtenidos en la CA}

En el desarrollo de la comunidad de aprendizaje (CA) se vivieron momentos que marcarían a cada una de las mujeres adolescentes participantes e indiscutiblemente al agente pedagógico., Se comparten los aprendizajes logrados, los conocimientos reforzados y develados, así como el sentir de las participantes al ser protagonistas en una experiencia de CA.

\section{Sensibilización}

Para esta pre-etapa de la ca se les propuso a las adolescentes una guía de preguntas sobre las cuales pudiesen intercambiar información con una de sus pares, y posteriormente hacer el ejercicio integradas en cuartetos: ¿cómo me llamo?, ¿cómo me gusta que me digan?, ¿cuántos años 
tengo?, ¿qué grado escolar curso?, ¿cuál es mi pasatiempo favorito?, ¿qué cosas me agradan y cuáles me desagradan?, ¿qué me motiva a venir a este taller?

En función de las respuestas emitidas por las participantes, la facilitadora les preguntó si identificaban similitudes y diferencias en sus respuestas, a lo que contestaron que Sí. Posteriormente, se les volvió a preguntar a qué creían ellas que se debían esas similitudes y diferencias. A continuación, las respuestas emitidas:

\begin{tabular}{|c|c|}
\hline SIMILITUDES & DIFERENCIAS \\
\hline $\begin{array}{l}\checkmark \text { Somos adolescentes. } \\
\checkmark \text { Pertenecemos a esta comunidad. }\end{array}$ & $\begin{array}{ll}\checkmark & \text { No todas somos iguales. } \\
\checkmark & \text { No todas pensamos igual. } \\
\checkmark & \text { No todas tenemos la misma vida. } \\
\checkmark & \text { Aunque adolescentes, tenemos } \\
& \text { edades diferentes. }\end{array}$ \\
\hline
\end{tabular}

Aprovechando la dinámica lograda, y en virtud de que se les observaba muy conversadoras y sociables en la actividad de binas y cuartetos, se volvió a cuestionar a las adolescentes, respecto a la comunicación con sus padres, particularmente, si así hablaban con ellos, si había fluidez. Las adolescentes respondieron recurrentemente que no, y las razones fueron: "no hay confianza" y "no hay la libertad de opinar".

Después de apreciar las respuestas de las adolescentes a los cuestionamientos hechos, es interesante, en la primera parte, la precisión al identificar las causas de las diferencias en sus respuestas. De esa forma las adolescentes ponen a la luz principios teóricos que ellas de manera sorprendente reconocen y ponen en 92 juego en la práctica: el sujeto es único e irrepetible; el sujeto y su subjetividad a nivel pensamiento, realidad y experiencia.
En la segunda parte, cuando se les pregunta sobre la comunicación con sus padres, de igual manera se hace manifiesta una realidad vivida por las adolescentes de la comunidad. No obstante aquí entra la incertidumbre y se genera la pregunta de si eso pasa sólo con mujeres adolescentes o también con hombres adolescentes, puesto que en el taller solo participaron mujeres.

\section{Toma de decisión}

Otra de las actividades en la pre-etapa de la CA consistió en sondear entre las adolescentes, qué idea o noción tenían sobre comunidad y aprendizaje. Las respuestas versaron sobre: 


\begin{tabular}{|c|c|}
\hline COMUNIDAD & APRENDIZAJE \\
\hline $\begin{array}{l}\checkmark \text { Grupo de personas que se apoyan. } \\
\checkmark \text { Los que vivimos en un mismo en- } \\
\text { torno. }\end{array}$ & $\begin{array}{ll}\checkmark & \text { Conocer. } \\
\checkmark & \text { Apropiarnos de algo que no con- } \\
& \text { ocemos. } \\
\checkmark & \text { Lección. } \\
\checkmark & \text { Algo nuevo. }\end{array}$ \\
\hline
\end{tabular}

Posteriormente, y organizadas en dos equipos, las adolescentes se dieron a la tarea de integrar, y tomando como punto de partida las ideas anteriores, una definición de CA:

\begin{tabular}{|l|l|}
\hline EQUIPO 1 & EQUIPO 2 \\
\hline $\begin{array}{l}\text { Es un grupo de personas que se apoyan para } \\
\text { apropiarse de algo que no se conoce o tener una } \\
\text { lección o también algo nuevo para compartir. }\end{array}$ & $\begin{array}{l}\text { Un grupo de personas que viven en un mismo } \\
\text { entorno y comparten todo como algo nuevo. }\end{array}$ \\
\hline
\end{tabular}

En un primer momento, las aportaciones realizadas por las adolescentes dan muestra, por una parte, del referente que se posee sobre "comunidad" y "aprendizaje"; respecto al primero, éste se vincula con grupo y la pertenencia a un espacio geográfico, en tanto que el segundo elemento es concebido como algo a lo que se asciende, que resulta desconocido y nuevo.

En un segundo momento, al fusionar los dos componentes ya mencionados, se aprecia en la elaboración de una definición, por parte de las adolescentes, de comunidad de aprendizaje, (CA) ciertos elementos comunes expuestos por Berlanga (2005, p. 2) sobre la definición que él propone: "Es un espacio de relaciones entre personas que comparten sentidos y significados de mundo de vida, reunidos a partir de intereses compartidos para resolver necesidades de aprendizaje que den lugar a una práctica intencionada de transformación de su realidad".

En virtud de lo anterior, puede sostenerse que las adolescentes generaron una apreciación de cA aceptable, haciendo uso única y exclusivamente de sus conocimientos previos y del intercambio de los mismos. Respecto al proceso, en la construcción de nuevos aprendizajes, puede decirse también, que dicha experiencia pudo poner en juego lo señalado por Torres (1999), en torno a las CA:

Cada miembro de la comunidad es poten93 cialmente un educador y un educando. 
La educación y los aprendizajes son responsabilidad de todos y de cada cual.

La cooperación y la solidaridad deben permear no sólo en la supervivencia, sino en lo cultural y educativo.

La educación no se realiza sólo en el sistema escolar

Se valora el aprendizaje intergeneracional y entre pares.

\section{El sueño}

Otras dimensiones abordadas en la ca se articularon con las siguientes preguntas: ¿cuáles son las cosas que me gustaría hacer en la vida?, ¿pienso que puedo lograrlas?, ¿cómo es un día de mi vida? A continuación, se bosquejan las respuestas compartidas por las adolescentes.

Tabla 1. Preguntas y respuestas de las adolescentes sobre sus sueños

\begin{tabular}{|c|c|}
\hline PREGUNTA & RESPUESTA \\
\hline $\begin{array}{l}\text { ¿Cuáles } \\
\text { son las co- } \\
\text { sas que me } \\
\text { gustaría } \\
\text { hacer en la } \\
\text { vida? }\end{array}$ & $\begin{array}{l}\text { - } \quad \text { Ser feliz, y saber cosas nuevas. } \\
\text { - } \quad \text { Un futuro feliz y una familia. } \\
\text { - } \quad \text { Mejorar en mis estudios. } \\
\text { - } \quad \text { Tener una familia y ser feliz. } \\
\text { - } \quad \text { Tener muchos lujos y ser feliz. }\end{array}$ \\
\hline \multicolumn{2}{|c|}{$\begin{array}{l}\text { ¿Pienso que puedo lograrlas? } \\
\text { Todas las adolescentes dijeron que "sí" pensaban que podrían lograrlo. Entre los argu- } \\
\text { mentos expuestos se destacan: } \\
\text { - Porque tengo la capacidad. } \\
\text { - Esforzándonos, sería posible. } \\
\text { - Con ayuda de mi familia. } \\
\text { - Podemos tener lo que queremos. } \\
\text { - Si estudio y me esfuerzo. }\end{array}$} \\
\hline $\begin{array}{l}\text { ¿Cómo es } \\
\text { un día de } \\
\text { tu vida? } \\
\text { Descríbelo }\end{array}$ & $\begin{array}{l}\text { - "Antes de irme a la escuela, debo dejar limpia la cocina y mi cuarto. Mi } \\
\text { mamá me lleva a la escuela en el auto. Estoy en la escuela, donde no } \\
\text { siento mucha aceptación, porque soy muy inteligente. Mamá va por } \\
\text { mí a la escuela, comemos, hago mi tarea. Me voy a mi cuarto, porque } \\
\text { no me gusta estar con mi hermanita, me aburre. Veo tele hasta tarde" } \\
\text { (palabras de una adolescente que compartió este testimonio). }\end{array}$ \\
\hline
\end{tabular}


¿Cómo es un día de tu vida? Descríbelo
- "Me levanto a las 5:30 de la mañana, porque entro a las 7:00 a mi escuela. Mi mamá o yo me preparo algo para desayunar. Me visto y tomo la combi que me deja en la escuela. En la escuela tengo muchos amigos(as) y me divierto. Al salir de la escuela me voy al trabajo de mi mamá y le ayudó en la limpieza de la escuela, y al terminar nos venimos juntas a casa, comemos juntas, luego hago mi tarea, me baño. A veces voy a la iglesia a servir porque soy monaguillo. Veo tele y me voy a dormir" (palabras de una adolescente que compartió este testimonio) En la primera ronda de participaciones, y siendo la última para hacer su intervención, una adolescente, preguntó a la facilitadora: ¿puedo hacerlo diferente? La facilitadora dijo "sí, adelante".

- $\quad$ "En mi casa hay mucha violencia intrafamiliar. Mi hermana pelea mucho con mi mamá y con mi papá. Luego mis papás le pegan mucho a ella, y de paso a mí también. Yo le digo a mi hermana que se porte bien. Además, va mal en la escuela, no hace las tareas. Como mi mamá también ya empezó a trabajar, nos quedamos solas en la casa, y nadie nos revisa las tareas. Una vez mi hermana amenazó a mi mamá con un cuchillo, y ese día mi papá y mi mamá le pegaron muy feo. Ella les gritó que se iba a ir de la casa.

Una noche, no estaba ni mi mamá ni mi papá, y mi hermana me dijo que nos fuéramos, que ella tenía dinero, y me enseñó unos billetes. Yo le dije que no. Ella insistía que nos fuéramos con mi tía, una hermana de mi papá, porque en esa casa nadie nos quería.

Me gustaría que hablaras con mi hermana, porque yo no quiero que le sigan pegando o que un día se vaya de la casa".

En el grupo se guardó un gran silencio y el resto de las adolescentes se mantenían quietas en tanto su compañera daba su testimonio. Una de ellas (la de mayor edad en ese momento) se puso de pie y abrazó a su compañera que empezaba a llorar. Después ninguna de ellas habló.

La facilitadora, reactivó la participación diciendo: su compañera ha tenido el valor de compartir estas experiencias de su vida. Recuerden los valores de esta CA, y sobre todo les pido que lo que aquí se comparte y se diga aquí se queda.

Posteriormente la facilitadora se dirigió a la participante del testimonio y le dijo que contaría con su ayuda y que se intentaría hablar con su hermana tan pronto como fuera posible. Se le agradeció la apertura y la confianza para con el grupo. 


\begin{tabular}{|c|c|}
\hline $\begin{array}{l}\text { ¿Cómo es } \\
\text { un día de } \\
\text { tu vida? } \\
\text { Descríbelo }\end{array}$ & $\begin{array}{l}\text { Seguidamente, una adolescente tomó la palabra y mencionó de un } \\
\text { centro de ayuda del que se dispone en el municipio para apoyar a } \\
\text { las familias con problemas. El resto, ofreció palabras de aliento a su } \\
\text { compañera, y externaron que era una situación difícil, que no se imag- } \\
\text { inaban que ella y su hermana vivieran en esa violencia. }\end{array}$ \\
\hline
\end{tabular}

Fuente: elaboración propia.

Las respuestas de las adolescentes a las dos primeras preguntas reflejaron sus máximas aspiraciones para un futuro no muy lejano: "ser felices". Sin embargo, surgen ahora otras interrogantes, como por ejemplo ¿qué es para ellas ser felices o qué es la felicidad?, ¿la felicidad es algo que se logra?, ¿en función de qué se logra? De igual manera, surge la inquietud por pensar de si en su etapa de adolescentes ison o si se sienten felices o no? Sin duda, estas preguntas, pueden servir para ser contempladas en una segunda experiencia con el grupo de adolescentes o con otra población, y adoptarla como una estrategia para posteriores procesos de mayor análisis y reflexión.

Algo cautivante y digno de reconocer lo constituyó también la actitud expresa de las adolescentes ante la vida, la confianza en sí mismas y en sus propias capacidades pues ello, se traduce en una enorme posibilidad de no sólo querer, sino de poder ser diferentes.

Con el cuestionamiento ¿cómo es un día de mi vida?, se colocaron "sobre la 96 mesa" un mosaico de realidades propias que viven cotidianamente cada una de las adolescentes. Este ejercicio, permitió tener un significativo acercamiento con las adolescentes. Evidentemente, el compartir los testimonios generó una dinámica diferente en el taller, permitió que la CA se instaurara en otra faceta, que se experimentaran otras formas de entrar en relación con el "otro", de, solidaridad. de compartir un sentimiento, una situación e incluso reflexionar sobre la nuestra.

\section{Toma de decisiones}

Con el segundo bloque de preguntas, ¿qué es proyecto de vida?, ¿qué conlleva un proyecto de vida?, ¿qué es ser mujer o qué significa para mi ser mujer?, ¿qué tipo de mujeres queremos ser?, y ¿podríamos intentar ser diferentes?, la intención fue contrastar los roles tradicionales de las adolescentes hasta ese momento desempeñados y asumidos de manera natural con el que o a los que ellas aspiran, articulando esto último con la importancia de pensar y trabajar en un proyecto de vida. 
Tabla 2. Preguntas y respuestas de las adolescentes sobre toma de decisiones

\begin{tabular}{|c|c|}
\hline PREGUNTA & RESPUESTA \\
\hline $\begin{array}{l}\text { ¿Qué es } \\
\text { ser mujer } \\
\text { o qué } \\
\text { significa } \\
\text { para mí ser } \\
\text { mujer? }\end{array}$ & $\begin{array}{l}\text { Amor, bienestar, paz. } \\
\text { Amor, comprensión. } \\
\text { Ser alguien importante en la vida, amor, comprensión, paz. Ser mujer es muy } \\
\text { bonito. } \\
\text { Amor, ternura. } \\
\text { Cariñosa, amorosa, libre, amada, ternura. } \\
\text { Ser libre, que tengamos nuestros derechos. }\end{array}$ \\
\hline $\begin{array}{l}\text { ¿Qué } \\
\text { mujeres } \\
\text { queremos } \\
\text { ser? }\end{array}$ & $\begin{array}{l}\text { Una mujer feliz, alegre y divertida. } \\
\text { Un poco rica, tener una estabilidad económica. } \\
\text { Una mujer importante y ser rica. } \\
\text { Muy importante, ser alguien en la vida, tener una carrera. } \\
\text { Ser una mujer buena y ayudar a otros. } \\
\text { Ser buena, solidaria. }\end{array}$ \\
\hline $\begin{array}{l}\text { ¿Podríamos } \\
\text { intentar ser } \\
\text { diferentes? } \\
\text { ¿Por qué? }\end{array}$ & $\begin{array}{l}\text { Sí, porque todas somos diferentes. } \\
\text { Sí, si se intenta sí. } \\
\text { Quizás, porque tal vez ahora no me comprenden o entienden. Mi familia pro- } \\
\text { mete y no cumple. } \\
\text { Sí. } \\
\text { Sí. } \\
\text { Sí, porque quiero ser alguien importante en la vida. }\end{array}$ \\
\hline $\begin{array}{l}\text { ¿Qué es un } \\
\text { proyecto } \\
\text { de vida? }\end{array}$ & $\begin{array}{l}\text { Es una meta. } \\
\text { Son sueños. } \\
\text { Es un camino. } \\
\text { Es un punto de vista a nuestro futuro. } \\
\text { Tomar decisiones. } \\
\text { Hacer las cosas bien. } \\
\text { Tener una dirección. }\end{array}$ \\
\hline $\begin{array}{l}\text { ¿Qué } \\
\text { conlleva o } \\
\text { implica un } \\
\text { proyecto } \\
\text { de vida? }\end{array}$ & $\begin{array}{l}\text { Ser constantes. } \\
\text { No rendirse. } \\
\text { Ser positivos. } \\
\text { Aprender a trabajar en equipo. } \\
\text { Esforzarse. } \\
\text { Apoyarse unos a otros. } \\
\text { Que exista la comunicación. }\end{array}$ \\
\hline
\end{tabular}

Fuente: elaboración propia. 
Para las adolescentes ser mujer se tradujo principalmente en: amor, ternura y comprensión. En menor intensidad, aunque no por ello menos importante, para otras participantes ser mujer les evocó: libertad y derechos. Revisando y reflexionando sobre las respuestas a esta pregunta, se hace notar no solo la acepción o significado que predomina entre las adolescentes respecto a ser mujer, sino que también puede derivarse que dicha acepción influye o puede influir en la auto-determinación de sus roles tradicionales tanto en su edad actual como en sus vidas futuras. De igual manera, se corrobora la visión de mujer que social, cultural y religiosamente se ha cultivado y reforzado entre las generaciones y las familias, al menos en ese contexto. Sin duda, es grato y significativo también toparse con las segundas respuestas, que en definitiva vienen a constituir, no únicamente, una apreciación distinta, sino toda una alternativa, una actitud, una visión, una colocación más democrática de ser mujer, dicho y manifiesto no por otros, sino por quienes encarnan esa condición de ser mujer, he allí la riqueza y relevancia del pensar y sentir manifiesto por las adolescentes.

En las siguientes dos preguntas, que se complementan entre sí, llama la atención las respuestas dadas a la penúltima de ellas, pues las apreciaciones respecto a qué mujer se quiere ser resultan contundentes y precisas entre las adolescentes, 98 no así sus respuestas para la última pregunta, ya que aun cuando se dice que sí se puede ser diferente, pareciera que aún no hay seguridad o elementos suficientes para decir por qué, lo cual es muy comprensible y refleja la edad propia de las adolescentes; sin embargo, haciendo otra lectura, ese "sí" puede traducirse, al mismo tiempo, en una respuesta que refleja el valor, que puede estar gestándose en un desafío, en una $\mathrm{fe}$, en una esperanza o en una alternativa para estas valiosas mujeres adolescentes que dicen que sí se puede ser diferente.

Con respecto a las preguntas que aluden a un proyecto de vida, las adolescentes en su mayoría lograron construir una respuesta acertada, aun cuando hasta ese momento no se les había facilitado nada de información al respecto. También fueron muy significativas las palabras empleadas por las adolescentes para indicar lo que en un momento dado implicaría la consecución de un proyecto de vida. Finalmente, con esta última fase de la ca la intención fue la posibilidad de repensarse, de ver la vitalidad de los propios sueños y la generación de estrategias para materializarlos y reorientarlos, en este caso a través de un proyecto de vida.

\section{Conclusiones y recomendaciones}

Vivir la experiencia de gestar y coordinar la comunidad de aprendizaje (CA) se tradujo en una grandiosa, significativa, transformadora y grata experiencia educativa-formativa, pero al mismo tiempo se tornó en un gran reto. 
Finalmente, el cometido inicial se logró. En primer lugar, las mujeres adolescentes participantes se aproximaron a su forma de pensar, de sentir y de actuar, así como a develar las razones por las que lo hacen de una u otra manera. En segundo lugar, las participantes tuvieron la apertura para analizar y conocer sus experiencias personales, su historia, su pasado, y la formación como las personas que son actualmente, de modo que puedan entender mejor su desarrollo y cómo esto influye en su presente y futuro. En tercer lugar, el ejercicio de sociabilizar el proceso de construcción de un proyecto de vida para rememorar el proceso a iniciativa de las participantes; en tercer lugar, sensibilizar a las mujeres sobre la importancia de repensarse de otro modo como mujeres, la vitalidad de los propios sueños y la generación de estrategias para materializarlos y reorientarlos.

El proceso acontecido y los resultados obtenidos en la CA, permiten esbozar conclusiones respecto a que:

Una CA tiene un inicio, pero no un término,

Una ca constituye un espacio de aprendizajes de diversa naturaleza, de expresión, de encuentros y de experiencias inusitadas.

Participar de la coordinación de una CA no solo demanda poseer conocimientos sobre lo que se delimite trabajar, sino, sobre todo, mucha apertura, confianza, credibilidad, paciencia, y habilidades para la escucha y la empatía.

No perder de vista el objetivo que persigue la CA, y recordarlo con regularidad entre los participantes para que todos tengamos claridad y conocimiento del punto de llegada.

Emplear una comunicación al alcance de la comprensión de los participantes.

Iniciada la CA, ir evaluando su desarrollo en lo grupal, a fin de realizar las adecuaciones necesarias.

Trabajar una CA con poblaciones no cautivas, genera incertidumbre; sin embargo, la CA puede convertirse en un espacio para generar-propiciar autorregulación entre los participantes y superar el "viejo" esquema de asistir por presión u obligación.

En una ca nadie sabe más que otro, sino que todos aprenden de todos.

La CA da la oportunidad para potenciar los saberes de los participantes, y reconocer que cada cual tiene algo valioso que compartir.

En suma, los resultados obtenidos en la CA constituyen una evidencia de los trabajos realizados a su interior, no obstante, el proceso vivido en compañía de las adolescentes que aceptaron el reto de participar en la CA, se queda grabado en la memoria y en el corazón de todas y cada una de nosotras. 


\section{Referencias}

Ávila, L. P. (2009). Manual de proyecto de vida completo. Recuperado de http://es.scribd. com/doc/13638628/Manual-de-Proyecto-de-Vida-Completo

Basis, H. (1996). Maestros ¿Formar o transformar? (E. Giordano, trad.). (2. ${ }^{a}$ Ed.). España: Gedisa, SA.

Berlanga, G. B. (2005). La educación como relación pedagógica para la resignificación del mundo de vida: en la propuesta educativa de las comunidades de aprendizaje. Revisión de la comunidad de aprendizaje (CESDER). Recuperado de http://www.ucired.org.mx/component/k2/ item/12-comunidades-aprendizaje/12-comunidades-aprendizaje

Cubero, R. (2005). Perspectivas constructivistas. La intersección entre el significado. La interacción y el discurso. España: GRAÓ.

De Lella, C. (2003). Formación docente: El modelo hermenéutico-reflexivo y la práctica profesional. Recuperado de http:// bibliotecadigital.conevyt.org.mx/servicios/hemeroteca/decisio/d5/sab3.htm

Flores, S. S. (2012). Las comunidades de aprendizaje como mecanismo eficiente para la formación de formadoresen lasociedad del conocimiento. Revista CongresoUniversidad, I (2), 1-10. Recuperado de http://www. congresouniversidad.cu/revista/index.php/ congresouniversidad/article/view/91

Freire, P. (1994). La educación como práctica de la libertad. (L. Ronzoni, trad.). (42. ${ }^{\text {a }}$ Ed.). México: Siglo XXI.

Freire, P. (1999). Pedagogía del oprimido. (J. Mellado, trad.). (52. ${ }^{a}$ Ed.). México: Siglo XXI.

Gómez, A. J. (s.f.). Comunidades de Aprendizaje. Madrid, España: Centro de In- vestigación Social y Educativa (CREA). Recuperado de http://www.nodo50.org/ movicaliedu/comunaprendizaje.htm

Martínez, M. L. (2011). Notas y apuntes del curso propedéutico del Doctorado Formación de sujetos y conciencia histórica. San Cristóbal de las Casas, México: Instituto Pensamiento y Cultura en América Latina (IPECAL).

Pansza, G, M., Pérez, J. E. y Morán, O. P. (1992). Fundamentación de la didáctica. (Vol. I). (5. ${ }^{a}$ Ed.). México: Gernika.

Sosa, G. M. (2002). El taller: estrategia educativa para el aprendizaje significativo. (2. ${ }^{a}$ Ed.). Colombia: Círculo de Lectura Alternativa.

Torres, R. M. (1999). Comunidad de aprendizaje: una comunidad organizada para aprender. Documento presentado en el Seminario de Educación Integral: Articulación de Proyectos y Espacios de Aprendizaje. São Paulo, Brasil: CENPEC. Recuperado de http://aler.org/index. php?option=com_phocadownload\&view=file\&rid=20: comunidad-de-aprendizaje\&Itemid=220\&lang=es

Torres, R. M. (2001). Comunidad de aprendizaje. Repensando lo educativo desdeel desarrollo local y desde el aprendizaje. Documento presentado en el Simposio Internacional sobre Comunidades de Aprendizaje. Barcelona, España: Fórum-2004. Recuperado de http://www. rsu.uninter.edu.mx/doc/marco_conceptual/RepensandoloEducativodesdeelDesarrolloLocal.pdf 